\title{
Trajectories of Suicide Ideation, Nonsuicidal Self-Injury, and Suicide Attempts in a Nonclinical Sample of Military Personnel and Veterans
}

Craig J. Bryan, Psyd, ABPP, AnnaBelle O. Bryan, BSPH, Alexis M. May, MA, and E. David KLONSky, PhD

\begin{abstract}
Nonsuicidal self-injury (NSSI) is a risk factor for suicide attempts, but little is known about NSSI among military personnel and veterans, or about the temporal sequencing of NSSI relative to suicide ideation and attempts. This study evaluates trajectories of suicide ideation, NSSI, and suicide attempts in a sample of 422 military personnel and veterans. Of those with a history of NSSI, $77 \%$ also experienced suicide ideation. Suicide ideation emerged before NSSI $(67 \%)$ more often than the reverse $(17 \%)$. Of those with a history of suicide attempt, $41 \%$ also engaged in NSSI. NSSI emerged prior to the first suicide attempt $(91 \%)$ more often than the reverse (9\%). The length of time from suicide ideation to suicide attempt was longer for those who first engaged in NSSI (median $=3.5$ years) compared with those who did not engage in NSSI (median $=0.0$ years), Wald $\chi^{2}(1)=11.985, p=.002$. Age of onset was earlier for participants reporting NSSI only compared with those reporting both NSSI and suicide attempts (16.71 vs. 22.08 years), $F(1,45)=4.149, p=.048$. NSSI may serve as a "stepping stone" from suicide ideation to attempts for $41 \%$ of those who attempt suicide.
\end{abstract}

During the past decade, suicides and suicide attempts among U.S. military personnel and veterans have more than doubled (Ramchand, Acosta, Burns, Jaycox, \& Pernin, 2011). Although scientific studies focused on suicide attempts among military

Craig J. Bryan, National Center for Veterans Studies, The University of Utah, Salt Lake City, UT, USA; AnNaBelle O. Bryan, National Center for Veterans Studies, Salt Lake Community College, Salt Lake City, UT, USA; Alexis M. MaY and E. David Klonsky, Department of Psychology, The University of British Columbia, Vancouver, British Columbia, Canada. Address correspondence to Craig J. Bryan, National Center for Veterans Studies, The University of Utah, 260 S. Central Campus Dr., Room 205, Salt Lake City, UT 84112; E-mail: craig.bryan@utah.edu personnel and veterans have expanded during this time frame, relatively little is known about nonsuicidal self-injury (NSSI) in this population. NSSI entails behavior that is self-directed and deliberately results in injury or the potential for injury to oneself, without evidence of suicidal intent (Crosby, Ortega, \& Melanson, 2011). Studies suggest that prevalence rates of NSSI range from $4 \%$ to $6 \%$ in adults (Briere \& Gil, 1998; Klonsky, 2011; Klonsky, May, \& Glenn, 2013; Klonsky, Oltmann, \& Turkheimer, 2003 ) and up to $7 \%$ to $20 \%$ of adolescents and young adults (Klonsky et al., 2013; Whitlock, Eckenrode, \& Silverman, 2006). In clinical populations, NSSI is much more common, with $40 \%$ to $60 \%$ of adolescent psychiatric patients reporting a history 
of the behavior (Asarnow et al., 2011; DiClemente, Ponton, \& Hartley, 1991; Klonsky \& Muehlenkamp, 2007; Klonsky et al., 2013; Wilkinson, Kelvin, Roberts, Dubicka, \& Goodyear, 2011). Among U.S. military personnel and veterans, prevalence rates have been found to range from $4 \%$ to 14\% (Bryan \& Bryan, 2014; Klonsky et al., 2003), which is roughly comparable to nonmilitary populations.

Although NSSI and suicide attempts are similar in that both entail intentional, self-directed violence, NSSI is differentiated from suicide attempts based on their different motivations and intent. Specifically, suicide attempts include an explicit or implicit intent to die, whereas NSSI occurs without suicidal desire (Crosby et al., 2011). NSSI and suicide attempts also differ in that NSSI typically has higher prevalence rates, occurs with greater frequency, and typically involves less medically severe methods (Klonsky et al., 2013). Despite being conceptually and characteristically distinct from each other, however, NSSI and suicide attempts often co-occur. For instance, 63\% to $70 \%$ of adolescent psychiatric patients reporting a history of NSSI also report a history of suicide attempt (Asarnow et al., 2011; Nock, Joiner, Gordon, Lloyd-Richardson, \& Prinstein, 2006). NSSI has also been shown to have a robust relationship with suicide attempts. In four separate samples across community and clinical settings, NSSI had a medium to strong association with suicide attempt history. In fact, NSSI had a stronger association with suicide attempts than many other well-established suicide risk factors including depression, anxiety, and borderline personality disorder; only suicide ideation showed a stronger relationship with suicide attempts than NSSI (Klonsky et al., 2013). In prospective studies, a history of NSSI also predicts future suicide ideation and suicide attempts beyond the effects of other risk factors. For instance, Asarnow et al. found that a history of NSSI is associated with a fivefold increase in likelihood for a suicide attempt within the next 6 months among adoles- cents in outpatient psychiatric treatment, whereas a history of suicide attempts is associated with only a twofold increase in risk. In a community sample of adolescents, Guan, Fox, and Prinstein (2012) reported that NSSI was associated with a sevenfold increase in risk for future suicide attempts as compared to a ninefold increase associated with past suicide attempts. Among military personnel and veterans, NSSI that occurred prior to joining the military is associated with a threefold increase in severity of suicide ideation assessed several years later, while premilitary suicide attempts, by comparison, are associated with an eightfold increase in severity (Bryan \& Bryan, 2014).

Despite converging research supporting the relationship of NSSI with suicide attempts, little is known about how these distinct yet related behaviors emerge in relation to each other over time. One model for understanding the co-occurrence of NSSI with suicide attempts is the interpersonal-psychological theory of suicide (IPTS; Joiner, 2005), which posits that an individual must possess both the desire for suicide and the capability to inflict lethal self-injury in order to die by suicide or make a medically serious suicide attempt. Consistent with this model, there could be two potential pathways that explain the co-occurrence of NSSI and suicide attempts (Joiner, Ribeiro, \& Silva, 2012). In the first pathway, individuals first experience suicide ideation (i.e., desire for suicide) and then subsequently engage in NSSI, which habituates the individual to the fear of self-directed violence and the physical pain associated with such behavior, thereby increasing their capability for self-inflicted injury. NSSI can therefore function as an intermediary step to suicide attempt for certain individuals with suicide ideation. Consistent with the opponent process (Solomon, 1980), individuals who engage in repeated NSSI often experience positive emotional shifts afterward, thereby reinforcing the behavior (Chapman \& Dixon-Gordon, 2007). As fearlessness about self-directed violence and 
tolerance of physical pain are acquired over time, the individual's capability for engaging in medically serious suicide attempts increases. In the second possible pathway, individuals first engage in NSSI without suicidal intent and then subsequently experience the onset of suicide ideation. In this pathway, individuals begin with elevated capability for suicide via the same habituation and opponent processes described above and then develop the desire for suicide. In both pathways, NSSI is presumed to emerge before suicide attempts. To date, however, this specific assumption has not been tested.

Previous research has confirmed that suicide ideation most frequently emerges in the year before a suicide attempt (Kessler, Borges, \& Walters, 1999; Nock et al., 2008), but similar research focusing on NSSI and suicide ideation does not yet exist due in large part to the fact that most studies considering the co-occurrence of NSSI and suicide attempts did not assess or report age of onset for suicide ideation, NSSI, or suicide attempts (Asarnow et al., 2011; Guan et al., 2012; Klonsky et al., 2013; Nock et al., 2006). Our understanding of temporal trends among suicide ideation, NSSI, and suicide attempts is therefore limited. It also remains unknown how common each pathway is among individuals who engage in both NSSI and suicide attempts and how long it takes for individuals to transition from NSSI to suicide attempts. Previous findings suggest that the majority of individuals who make a suicide attempt do so within a year of the first onset of suicide ideation (Kessler et al., 1999; Nock et al., 2008), suggesting a relatively rapid transition from thought to action. Because these studies did not assess for NSSI, however, it is not possible to determine when (or whether) NSSI occurred relative to suicide ideation and attempts.

The primary aim of this study was to identify temporal sequences in suicide ideation, NSSI, and suicide attempts across the life span in a sample of military personnel and veterans who were enrolled in college classes. To address this aim, we sought to answer the following research questions:

1. Among those with a history of NSSI, what is the temporal relationship with suicide ideation onset?

2. Among those with a history of suicide attempt, what is the temporal relationship with NSSI onset?

3. How much time elapses between the first onset of suicide ideation, NSSI, and suicide attempts?

4. What is the mean age of onset for suicide ideation, NSSI, and suicide attempts?

\section{METHOD}

\section{Participants}

Participants were 422 military personnel and veterans enrolled in college classes at 4-year public $(n=227,53.8 \%)$, 4-year private $(n=40,9.7 \%), \quad 2$-year public $(n=104,24.7 \%)$, and for-profit online ( $n=44,10.4 \%)$ universities across the United States. Gender distribution was 294 (69.7\%) male, 115 (27.3\%) female, and 13 (3.1\%) unknown. Age ranged from 19 to 78 years $(M=36.29, \quad S D=10.25), \quad$ and racial distribution was 348 (82.5\%) Caucasian, 24 (5.7\%) African American, 14 (3.3\%) Native American, 11 (2.6\%) Asian, and 5 (1.2\%) Pacific Islander. Hispanic/Latino ethnicity was assessed separately and was endorsed by 47 (11.1\%) of participants. The majority of participants $(n=274,64.9 \%)$ were veterans no longer in military service (e.g., retired, honorably discharged); the remaining 148 (35.1\%) reported they were still serving in one of the military's components. Students represented all branches of service: 137 (32.5\%) Air Force, 156 (37.0\%) Army, 32 (7.6\%) Marines, 83 (19.7\%) Navy, and $5(1.2 \%)$ Coast Guard. Nearly three quarters $(n=305,72.3 \%)$ reported having been deployed at least once. 


\section{Procedures}

Participants were recruited from universities across the country by administrators working with student service members/ veterans on each campus (e.g., military and veteran student coordinators) during the 2013 calendar year (i.e., spring 2013, summer 2013, and fall 2013 academic terms). Invitations to partner on the project were sent to members of the National Association of Veteran's Program Administrators' e-mail listserv. Information regarding the study was then e-mailed to university coordinators who responded to this invitation and agreed to participate. These coordinators then forwarded an e-mail message containing a brief description of the project, an invitation to participate, and an embedded hyperlink for an online survey to student service members/veterans enrolled at their respective universities. Upon visiting the study Web site, student service members/ veterans reviewed the informed consent document and then proceeded to the survey if they agreed to participate. Upon completion of the survey, participants were invited to forward the hyperlink to the online survey to other student service members/ veterans. The survey was completed anonymously and took an average of 12 to 15 minutes to complete.

Quality checks were conducted by comparing the concordance of responding among similar items located throughout the survey, as well as checking for impossible or highly improbable values that might indicate careless responding (e.g., age of enlistment in military). There were no indictors of inconsistent responding or outliers that would adversely influence the data. Because of the methods employed, it is unknown what proportion of all student service members/veterans eligible to participate actually did so. However, 561 individuals accessed the survey Web site, of whom 437 (77.9\%) completed the survey. Of these 437, 15 $(3.3 \%)$ had never served in the military and were therefore excluded, leaving $422 \mathrm{stu}-$ dent service members/veterans (i.e., $75.2 \%$ of all individuals accessing the survey). Participants were not compensated for completing the survey. Approval for the current study was provided by the University of Utah institutional review board.

\section{Instrument}

The self-report version of the SelfInjurious Thoughts and Behaviors Interview (SITBI; Nock, Holmberg, Photos, \& Michel, 2007) was used to assess lifetime suicide ideation (i.e., "Have you ever had thoughts of killing yourself?"), NSSI (i.e., "Have you ever actually engaged in nonsuicidal self-injury, that is, purposely hurting yourself without wanting to die, for example by cutting or burning?"), and suicide attempt (i.e., "Have you ever made an actual attempt to kill yourself in which you had at least some intent to die?"). Participants who endorsed suicide ideation, NSSI, or suicide attempt were then asked to report their age (in years) when they first experienced each.

The SITBI has good test-retest reliability $(\kappa>.70)$ over a 6 -month period, and responses to the items have shown strong correspondence with other measures of suicidal thoughts $(\kappa=.59)$ and suicide attempts $(\kappa=.65)$.

\section{Data Analysis}

Univariate and multivariate mean comparisons were made using generalized linear modeling with robust maximum-likelihood estimation due to considerable skew in most variables. For dichotomous outcomes, logistic regression was used. Age and gender were selected as covariates, but did not influence outcomes; discussion of the results therefore does not explicitly reference these covariates. Data were missing from six (1.4\%) participants.

\section{RESULTS}

During their lifetime, 141 (33.4\%) participants reported experiencing suicide 
TABLE 1

Proportions of Military Personnel and Veterans Reporting Lifetime Incidence of Suicide

Ideation, Nonsuicidal Self-Injury (NSSI), and/or Suicide Attempt $(n=422)$

\begin{tabular}{|c|c|c|c|c|}
\hline & \multicolumn{4}{|c|}{ Suicide Ideation (\%) } \\
\hline & \multicolumn{2}{|l|}{ No } & \multicolumn{2}{|c|}{ Yes } \\
\hline & \multicolumn{2}{|c|}{ Suicide attempt } & \multicolumn{2}{|c|}{ Suicide attempt } \\
\hline & No & Yes & No & Yes \\
\hline \multicolumn{5}{|l|}{ NSSI } \\
\hline No & $270(64.0)$ & $\mathrm{n} / \mathrm{a}$ & 84 (19.9) & $16(3.8)$ \\
\hline Yes & 11 & $\mathrm{n} / \mathrm{a}$ & 28 (6.6) & $13(3.1)$ \\
\hline
\end{tabular}

ideation, $52(12.3 \%)$ reported engaging in NSSI, and $29(6.9 \%)$ reported a suicide attempt. Endorsed methods for NSSI included cutting/carving (78.7\%), hitting self $(44.7 \%)$, scraping the skin $(36.2 \%)$, burning $(34.0 \%)$, picking at the body $(29.8 \%)$, inserting sharp objects under the skin (25.5\%), and "other" (10.6\%). Endorsed methods for suicide attempts included over- dose on drugs or medications (65.5\%), strangling or asphyxiation (24.1\%), firearm/ gun $(10.3 \%)$, hanging $(10.3 \%)$, transportation related $(10.3 \%)$, scratching or cutting (10.3\%), stepping into traffic (6.9\%), jumping from a height $(6.9 \%)$, poison or caustic substance $(3.4 \%)$, burning (3.4\%), stabbing or puncturing $(3.4 \%)$, and drowning $(3.4 \%)$.

The overlap of suicide ideation, NSSI, and suicide attempts reported by participants is displayed in Table 1 . As can be seen, the largest subgroup $(n=270,64.0 \%)$ reported no self-injurious thoughts or behaviors of any kind. The next largest subgroups, in descending order, were suicide ideation only ( $n=84,19.9 \%)$; suicide ideation and NSSI $(n=28,6.6 \%)$; suicide ideation and suicide attempt $(n=16,3.8 \%)$; suicide ideation, NSSI, and suicide attempt $(n=13,3.1 \%) ;$ and NSSI only $(n=11$, $2.6 \%)$. To identify trajectories over time, we organized self-reported ages of onset for suicide ideation, NSSI, and suicide attempts in temporal order from earliest to latest. Nine trajectories were identified in the current sample (Table 2).

TABLE 2

Trajectories of Suicide Ideation, NSSI, and Suicide Attempt Among 422 Military Personnel and Veterans Enrolled in College

\begin{tabular}{|c|c|c|c|c|}
\hline Trajectory & $n$ & $\begin{array}{c}\text { Full } \\
(n=422) \\
\%\end{array}$ & $\begin{array}{c}\text { NSSI } \\
(n=52) \\
\%\end{array}$ & $\begin{array}{c}\text { SA } \\
(n=29) \\
\%\end{array}$ \\
\hline 0 No SITB & 275 & 65.2 & - & - \\
\hline $1 \mathrm{SI}$ & 84 & 19.9 & - & - \\
\hline $2 \mathrm{SI} \rightarrow \mathrm{SA}$ & 16 & 3.8 & - & 55.2 \\
\hline $3 \mathrm{SI} \rightarrow \mathrm{SA} \rightarrow \mathrm{NSSI}$ & 1 & 0.2 & 3.7 & 3.4 \\
\hline $4 \mathrm{SI} \rightarrow \mathrm{NSSI} \rightarrow \mathrm{SA}$ & 10 & 2.3 & 19.2 & 34.5 \\
\hline $5 \mathrm{SI} \rightarrow \mathrm{NSSI}$ & 13 & 3.1 & 25.0 & - \\
\hline 6 NSSI & 11 & 2.6 & 21.2 & - \\
\hline $7 \mathrm{NSSI} \rightarrow \mathrm{SI}$ & 6 & 1.4 & 11.5 & - \\
\hline $8 \mathrm{SI} / \mathrm{NSSI}^{\mathrm{a}}$ & 6 & 1.4 & 11.5 & - \\
\hline Missing data ${ }^{\mathrm{b}}$ & 6 & 1.4 & 7.9 & 6.9 \\
\hline
\end{tabular}

Note. Total $n$ missing for each subgroup was $n=6$ for full sample, $n=6$ for SI, $n=4$ for NSSI, and $n=2$ for SA.

SITB, self-injurious thoughts and behaviors; SI, suicide ideation; SA, suicide attempt; NSSI, nonsuicidal self-injury.

${ }^{\text {a}}$ Participants reported the onset of suicide ideation and NSSI during the same year, so temporal sequencing could not be determined.

${ }^{\mathrm{b}} \mathrm{Six}$ participants did not report age of onset for one or more forms of SITB. 
Among Those with a History of NSSI, for What Proportion Did First Suicide Ideation Precede First NSSI?

Of the 52 participants with a history of NSSI, complete data were available from 47 (90.4\%). The majority of participants with a history of NSSI $(n=36,76.6 \%)$ also experienced suicide ideation at some point during their lives (Trajectories 3, 4, 5, 7, and 8). Of these 36 participants, most $(n=24,66.7 \%)$ reported the onset of suicide ideation before the onset of NSSI (Trajectories 3, 4, 5) and six (16.7\%) reported the onset of NSSI before first experiencing suicide ideation (Trajectory 7). The temporal sequence could not be determined for six $(16.7 \%)$ participants because they reported onset of suicide ideation and NSSI during the same year (Trajectory 8). Results indicate that suicide ideation emerged before NSSI approximately four times more often than the reverse.

\section{Among Those with a History of Suicide Attempt, for What Proportion Did First NSSI Precede First Suicide Attempt?}

Of the 29 participants with a history of suicide attempt, complete data were available from 27 (93.1\%). Just under half of this subgroup $(n=11,40.7 \%)$ also engaged in NSSI (Trajectories 3 and 4). For those who reported both NSSI and suicide attempts, almost all $(n=10,90.9 \%)$ reported the onset of NSSI prior to the first suicide attempt (Trajectory 4); only one $(9.1 \%)$ participant reported their first suicide attempt occurred prior to the onset of NSSI (Trajectory 3). Results indicate that NSSI occurred before the first suicide attempt approximately 10 times more often than the reverse.

How Much Time Elapses Between the First Onset of Suicide Ideation, NSSI, and Suicide Attempts?

To determine the mean length of time to transition from one form of self-directed violence to another, we calculated the differ- ences between the ages of onset for suicide ideation, NSSI, and suicide attempt within each trajectory, and then aggregated across multiple trajectories. For example, to determine the length of time for suicide ideation to transition to suicide attempt, we subtracted the age of onset for suicide ideation from the age of onset for suicide attempt for each of the four trajectories in which suicide ideation preceded suicide attempts (i.e., Trajectories 2, 3, 4, and 5). We then calculated the mean across all four of these trajectories to obtain the overall mean length of time to transition from first onset of suicide ideation to first instance of suicide attempt. For Trajectory 8 , which was comprised of six participants who reported the onset of suicide ideation and NSSI during the same year of their lives, the difference between ages of onset was assigned a value of 0 . Because the temporal sequencing of these six participants could not be determined, participants in Trajectory 8 were included in the calculation of the mean transition time from suicide ideation to NSSI transition as well as the mean transition time from NSSI to suicide ideation.

As summarized in Table 3, the median length of time from first onset of suicide ideation to suicide attempt was less than 1 year $\quad($ median $=0.0, \quad M=3.96, \quad S D=$ 6.55). For those who engaged in NSSI prior to their suicide attempt, the transition from suicide ideation to suicide attempt was approximately 3.5 years longer (median $=$ 3.5, $M=6.60, S D=7.52$ ) than for those who transitioned directly from suicide ideation to suicide attempt (median $=0.0$, $M=2.56, S D=5.73)$. This difference was statistically significant, Wald $\chi^{2}(1)=$ $11.985, p=.002$. Those who engaged in NSSI were significantly less likely to transition from suicide ideation to suicide attempt within 1 year than those who did not engage in NSSI $(54.5 \%$ vs. $75.0 \%$; $\mathrm{AOR}=.14[.02$, .97], $p=.047)$. Results suggest that, on average, individuals with suicide ideation who engage in NSSI before making a suicide attempt tend to take longer to transition from suicide ideation to suicide attempt than 
BRYAN ET AL.

TABLE 3

Length of Time (in Years) to Transition from One Form of Self-Directed Violence to Another

\begin{tabular}{lrcll}
\hline Trajectory & $n$ & $M$ & $S D$ & Median \\
\hline SI to SA & 27 & 3.96 & 6.55 & 0.0 \\
2 SI $\rightarrow$ SA & 16 & 2.56 & 5.73 & 0.0 \\
3 SI $\rightarrow$ SA $\rightarrow$ NSSI & 1 & 0.00 & - & 0.0 \\
4 SI $\rightarrow$ NSSI $\rightarrow$ SA & 10 & 6.60 & 7.52 & 3.5 \\
SI to NSSI & 30 & 3.03 & 6.08 & 1.5 \\
3 SI $\rightarrow$ SA $\rightarrow$ NSSI & 1 & 1.00 & - & 1.0 \\
4 SI $\rightarrow$ NSSI $\rightarrow$ SA & 10 & 1.50 & 7.32 & 0.5 \\
5 SI $\rightarrow$ NSSI & 13 & 5.77 & 5.83 & 4.0 \\
8 SI/NSSI & 6 & 0.00 & 0.00 & 0.0 \\
NSSI to SI & 12 & 1.17 & 2.25 & 0.5 \\
7 NSSI $\rightarrow$ SI & 6 & 2.33 & 2.80 & 1.0 \\
8 SI/NSSI & 6 & 0.00 & 0.00 & 0.0 \\
NSSI to SA & 10 & 5.10 & 5.82 & 4.5 \\
4 SI $\rightarrow$ NSSI $\rightarrow$ SA & 10 & 5.10 & 5.82 & 4.5 \\
\hline
\end{tabular}

SI, suicide ideation; SA, suicide attempt; NSSI, nonsuicidal self-injury.

individuals who transition directly from suicide ideation to suicide attempt.

The transition from suicide ideation to NSSI took approximately 1.5 years (median $=$ $1.5, M=3.03, S D=6.08$ ). For those who engaged in both NSSI and suicide attempts, the transition from suicide ideation to NSSI was faster (median $=0.5, M=1.50, S D=$ 7.325) than for those who only engaged in NSSI (median $=4.0, M=5.77, S D=5.83$ ). This difference approached statistical significance, Wald $\chi^{2}(1)=2.906, p=.088$. In contrast, the transition from NSSI to suicide ideation occurred in less than 1 year (median $=0.5, M=1.17, S D=2.25)$, suggesting the transition from NSSI to suicide ideation occurs more rapidly than the transition from suicide ideation to NSSI.

For those participants who first engaged in NSSI and then made a suicide attempt, the transition from NSSI to suicide attempt took approximately 4.5 years (median $=4.5, M=5.10, S D=5.82$ ).

What is the Mean Age of Onset for Suicide Ideation, NSSI, and Suicide Attempts?

We next classified participants into three groups according to history of NSSI and suicide attempt: NSSI only $(n=39)$, NSSI and suicide attempt (NSSI + SA, $n=$ $13)$, and suicide attempt only (SA; $n=16$ ). Suicide ideation emerged approximately 4 years earlier among participants in the NSSI group as compared to the NSSI + SA and SA group, but this difference was not statistically significant, $\quad F(2, \quad 52)=1.994$, $p=.146$, partial $\eta^{2}=.071$. The NSSI group also reported a significantly earlier age of onset for NSSI than the NSSI + SA group, $F$ $(1,45)=4.149, p=.048$, partial $\eta^{2}=.084$. There were no differences in age of onset for suicide attempts between the NSSI + SA and SA groups, Wald $\chi^{2}(1)=0.928, p=.335$. Results are summarized in Table 4.

\section{DISCUSSION}

In the current study we aimed to identify temporal sequences and trajectories of self-injurious thoughts and behaviors in a sample of military personnel and veterans who were enrolled in college courses. Results not only provide information about general trends in the emergence of NSSI and suicide attempts over time, but also suggest the possible existence of different subgroups of individuals who engage in 
TABLE 4

Mean Age of Onset for Suicide Ideation, NSSI, and Suicide Attempt Among Military Personnel and Veterans with a History of NSSI Only, NSSI and Suicide Attempt, and Suicide Attempt Only

\begin{tabular}{|c|c|c|c|c|c|c|}
\hline \multirow[b]{2}{*}{ Age of onset, years } & \multicolumn{2}{|c|}{ NSSI $(n=39)$} & \multicolumn{2}{|c|}{$\mathrm{NSSI}+\mathrm{SA}(n=13)$} & \multicolumn{2}{|c|}{$\mathrm{SA}(n=16)$} \\
\hline & $n$ & $M(S D)$ & $n$ & $M(S D)$ & $n$ & $M(S D)$ \\
\hline SI & 27 & $15.11(5.56)$ & 12 & $19.08(10.50)$ & 15 & $18.88(6.57)$ \\
\hline NSSI & 34 & $16.71(6.14)^{a}$ & 12 & $22.08(11.73)^{\mathrm{a}}$ & - & - \\
\hline SA & - & - & 11 & $24.82(10.31)$ & 15 & $21.44(7.76$ \\
\hline
\end{tabular}

Note. SI, suicide ideation; NSSI, nonsuicidal self-injury; SA, suicide attempt. ${ }^{\mathrm{a}} p=.048$.

self-directed violence. In terms of the temporal sequencing of self-directed violence over time, nine different trajectories were identified, the most common of which (20\% of the full sample, $59.6 \%$ of those with a history of any self-directed violence) was to experience suicide ideation without engaging in NSSI or suicide attempts. This finding suggests that the majority of participants who thought about suicide at some point in their lives had not (yet) progressed to self-directed violence at the time of data collection. Only 19\% of participants who experienced suicide ideation eventually went on to make a suicide attempt, a finding that is comparable to general population estimates, which range from $20 \%$ to $29 \%$ (Kessler et al., 1999; Nock et al., 2008). We also found that only $19 \%$ of participants who engaged in NSSI subsequently made a suicide attempt, which is generally consistent with previous studies reporting follow-up suicide attempt rates ranging from $13 \%$ to $53 \%$ among adolescent psychiatric samples (Asarnow et al., 2011; Wilkinson et al., 2011). Similar to these previous findings, our results suggest that NSSI was comparable to or better than suicide ideation as a risk factor for later suicide attempts in this sample.

In terms of trajectories among suicide ideation, NSSI, and suicide attempts over time, several general trends were noted. First, we found that NSSI occurred before suicide attempts in 10 of 11 (91\%) cases, whereas suicide attempts occurred before
NSSI in only 1 of $11(9 \%)$ cases. This suggests that NSSI almost always emerged before suicide attempts among our participants. Second, we found that suicide ideation emerged prior to the onset of NSSI in 24 of $36(67 \%)$ cases, whereas NSSI occurred prior to the onset of suicide ideation in only 6 of $36(17 \%)$ cases. Unfortunately, another 6 of $36(17 \%)$ participants reported the onset of suicide ideation and NSSI during the same year, thereby restricting our ability to clearly determine their temporal sequence. This pattern suggests that, in the current sample, it was more likely that NSSI emerged after suicide ideation. Taken together, these findings suggest that a more common pathway for the co-occurrence of NSSI and suicide attempts is to begin with suicide ideation, then to engage in NSSI as an intervening step, and finally to make a suicide attempt. This general trajectory from suicide ideation to NSSI to suicide attempt accounted for $37 \%$ of all suicide attempt cases, suggesting it is relatively common for NSSI to serve as a "stepping stone" to suicide attempts among those with suicide ideation, which is generally consistent with the IPTS's (Joiner, 2005) proposition that NSSI facilitates the acquisition of the capability for suicide among those who already desire suicide.

However, not all ideators who selfinjured progressed to attempt. In fact, a similar proportion of participants who engaged in NSSI after suicide ideation did 
not subsequently attempt suicide. This may suggest that NSSI sufficiently functioned as an emotion regulation strategy such that the desire to attempt abated. Previous research suggests that the most common motivation for NSSI is relief of emotional distress, being endorsed by slightly more than half of NSSI cases (Nock \& Prinstein, 2004). NSSI can also result in a visible and sometimes alarming marker of distress, such that service provision or social support may increase after NSSI, whether that function was intended or not (Nock, 2008). Thus, some ideators with NSSI may begin to receive mental health treatment and not continue down a trajectory toward attempt. It is also possible that some ideators and self-injurers had not yet made a suicide attempt at the time of participation in this study, but a suicide attempt will eventually occur at some point in the future. Prospective studies are needed in the future to control for this limitation.

For some participants, NSSI may have been insufficient as an emotion regulation strategy or may not result in treatment or support, and instead may facilitate the transition to suicide attempt. This might account for the observed differences in length of transition time from suicide ideation to suicide attempts between those with and without NSSI as an intervening step. In the current sample, the median length of time to transition from suicide ideation to suicide attempt was less than 1 year, which is consistent with prior research in the U.S. general population (Kessler et al., 1999; Nock et al., 2008). Interestingly, this transition time was significantly longer (median $=3.5$ years) for those who engaged in NSSI as an intermediate step than for those who transitioned directly from suicide ideation to suicide attempt (median $=0.0$ years). Furthermore, participants were more likely to transition from suicide ideation to suicide attempt in less than 1 year if they did not engage in NSSI as an intermediary step $(75 \%)$ than if they did $(55 \%)$. Taken together, the current findings suggest that NSSI may have "slowed down" the transition from suicide ideation to attempt in our sample, which expands on previous findings considering trajectories from suicidal thoughts to behaviors.

Overall results of the current study were not influenced by age and gender, although previous research suggests that NSSI is approximately twice as prevalent among female military personnel and veterans (Bryan \& Bryan, 2014). With regard to age, however, our finding that the age of onset for suicide ideation and NSSI was much earlier for the NSSI only group as compared to the SA only and NSSI + SA groups may suggest a developmental aspect to the emergence and trajectories of selfdirected violence. If replicated in other samples, this could potentially lead to more targeted interventions and prevention strategies.

From a clinical perspective, our results suggest there are several pathways from suicide ideation to suicide attempts, some of which are associated with relatively longer delays in the transition. Of note, over onethird of participants who made a suicide attempt engaged in NSSI first, suggesting NSSI may signal a prodromal stage of increasing risk for a substantial proportion of military personnel and veterans who make suicide attempts. Effective clinical intervention with those who engage in NSSI could prevent or derail the transition to suicide attempts, which could lead to a marked decrease in suicide attempts. It is critical to note that although NSSI is generally associated with a longer transition from suicide ideation to suicide attempt, nearly half of those with NSSI nonetheless transition from suicide ideation to suicide attempt in less than 1 year; clinicians and preventionists should therefore be quick to intervene as soon as possible after the initial onset of suicide ideation.

Conclusions based on the present study should be made cautiously in light of several limitations. First, our sample was comprised of military personnel and veterans who were enrolled in college classes, which might represent a relatively unique 
and/or select subset of military personnel and veterans that may not be representative of the larger population of military personnel and veterans. Although recent data suggest that rates of suicide ideation, NSSI, and suicide attempts are comparable among military and nonmilitary samples (Bryan, 2014; Bryan \& Bryan, 2014), our sample had relatively high rates of military-specific experiences (e.g., 72\% have deployed at least once) that may influence outcomes. For instance, previous research suggests that exposure to deployment-related stressors is associated with increased fearlessness about death and pain tolerance (Bryan \& Cukrowicz, 2011; Bryan, Cukrowicz, West, \& Morrow, 2010), both of which are proposed to contribute to the capability to engage in NSSI and make suicide attempts (Joiner, 2005). Generalization of results should therefore be made with caution until the present results can be replicated in additional samples. A second limitation of the current study is its retrospective, selfreport design, which may be vulnerable to recall bias and response bias. Future studies that utilize prospective designs are needed to confirm these findings, as are studies that use structured interviews administered by trained evaluators to ensure reliable assessment of self-directed violence and differentiation of NSSI from suicide attempt. Third, due to the relatively small size of some subgroups, we had limited power to further examine the associations among variables. Furthermore, in the present study we did not assess for the presence of psychiatric diagnoses, which are known risk factors for suicide ideation, suicide attempts, and NSSI, although previous research suggests that psychiatric disorders may have minimal influence on results (Asarnow et al., 2011; Klonsky et al., 2013; Nock et al., 2006). Additionally, the study did not measure the severity of NSSI or the lethality of attempts and thus was not able to assess the role of these variables in the relationship between NSSI and attempt. Despite these limitations, these results provide useful information regarding the relationships of suicide ideation, NSSI, and suicide attempt over time and suggest that greater attention to NSSI may be warranted for suicide prevention efforts, especially among high-risk populations such as military personnel and veterans.

\section{REFERENCES}

Asarnow, J. R., Porta, G., Spirito, A., Emslie, G., Clarke, G., Wagner, K. D., et al. (2011). Suicide attempts and nonsuicidal selfinjury in treatment resistant depression in adolescents: Findings from the TORDIA Study. Fournal of the American Academy of Child and Adolescent Psychiatry, 50, 772-781.

BrIERE, J., \& GIL, E. (1998). Self-mutilation in clinical and general population samples: Prevalence, correlates, and functions. American Fournal of Orthopsychiatry, 68, 609-620.

BRYAn, C. J. (2014). Sociodemographic correlates of suicidal thoughts and behaviors among college student service members/veterans. Unpublished manuscript.

Bryan, C. J., \& Bryan, A. O. (2014). Nonsuicidal self-injury among a sample of United States military personnel and veterans enrolled in college classes. Fournal of Clinical Psychology, 70, 874-885.

Bryan, C. J., \& Cukrowicz, K. C. (2011). Associations between types of combat violence and the acquired capability for suicide. Suicide and Life-Threatening Bebavior, 41, 126136.

Bryan, C. J., Cukrowicz, K. C., West, C. L., \& Morrow, C. E. (2010). Combat experience and the acquired capability for suicide. Journal of Clinical Psychology, 66, 1044-1056.

Chapman, A. L., \& Dixon-Gordon, K. L. (2007). Emotional antecedents and consequences of deliberate self-harm and suicide attempts. Suicide and Life-Threatening Behavior, 37, 543-552.

Crosby, A. E., Ortega, L., \& Melanson, C. (2011). Self-directed violence surveillance: Uniform definitions and recommended data elements, version 1.0. Atlanta, GA: Centers for Disease Control and Prevention.

DiClemente, R. J., Ponton, L. E., \& Hartley, D. (1991). Prevalence and correlates of cutting behavior: Risk for HIV transmission. Journal of the American Academy of Child and Adolescent Psychiatry, 30, 735-739. 
Guan, K., Fox, K. R., \& Prinstein, M. J. (2012). Nonsuicidal self-injury as a time-invariant predictor of adolescent suicide ideation and attempts in a diverse community sample. Fournal of Consulting and Clinical Psychology, 80, 842-849.

JoINER, T. E. (2005). Why people die by suicide. Cambridge: Harvard University Press.

Joiner, T. E., Ribeiro, J. D., \& Silva, C. (2012). Self-injury, suicidal behavior, and their co-occurrence as viewed through the lens of the interpersonal theory of suicide. Current Direction in Psychological Science, 21, 342-347.

Kessler, R. C., Borges, G., \& Walters, E. E. (1999). Prevalence of and risk factors for lifetime suicide attempts in the National Comorbidity Survey. Archives of General Psychiatry, 56, 617-626.

Klonsky, E. D. (2011). Non-suicidal self-injury in United States adults: Prevalence, sociodemographics, topography, and functions. Psychological Medicine, 41, 1981-1986.

Klonsky, E. D., May, A. M., \& GlenN, C. R. (2013). The relationship between nonsuicidal self-injury and attempted suicide: Converging evidence from four samples. Abnormal Psychology, 122, 231-237.

Klonsky, E. D., \& Muehlenkamp, J. J. (2007). Self-injury: A research review for the practitioner. Fournal of Clinical Psychology, 63, 1045-1056.

Klonsky, E. D., Oltmann, T. F., \& Turkheimer, E. (2003). Deliberate self-harm in a nonclinical population: Prevalence and psychological correlates. American Fournal of Psychiatry, 160, 1501-1508.

Nock, M. K. (2008). Actions speak louder than words: An elaborated theoretical model of the social functions of self-injury and other harmful behaviors. Applied and Preventive Psychology, 12, 159-168.

Nock, M. K., Borges, G., Bromet, E. J., Alonso, J., Angermeyer, A., Bruffaerts,
R., ET AL. (2008). Cross-national prevalence and risk factors for suicide ideation, plans, and attempts. The British Fournal of Psychiatry, 192, 98-105.

Nock, M. K., Holmberg, E. B., Рhotos, V. I., \& Michel, B. D. (2007). Self-injurious thoughts and behaviors interview: Development, reliability, and validity in an adolescent sample. Psychological Assessment, 19, 309-317.

Nock, M. K., Joiner, T. E., Gordon, K. H., Lloyd-Richardson, E., \& Prinstein, M. J. (2006). Non-suicidal self-injury among adolescents: Diagnostic correlates and relation to suicide attempts. Psychiatry Research, 144, 65-72.

Nock, M. K., \& Prinstein, M. J. (2004). A functional approach to the assessment of selfmutilative behavior. Fournal of Consulting and Clinical Psychology, 72, 885-890.

Ramchand, R., Acosta, J., Burns, R. M., Jaycox, L. H., \& Pernin, C. G. (2011). The war within: Preventing suicide in the U.S. military. Santa Monica, CA: RAND Corporation.

Solomon, R. L. (1980). The opponentprocess theory of acquired motivation: The costs of pleasure and the benefits of pain. American Psychologist, 35, 691-712.

Whitlock, J., Eckenrode, J., \& SilverMAN, D. (2006). Self-injurious behaviors in a college population. Pediatrics, 117, 1939-1948.

Wilkinson, P., Kelvin, R., Roberts, C., Dubicka, B., \& Goodyear, I. (2011). Clinical and psychosocial predictors of suicide attempts and nonsuicidal self-injury in the adolescent depression antidepressants and psychotherapy trial (ADAPT). American fournal of Psychiatry, 168, 495-501.

Manuscript Received: March 20, 2014

Revision Accepted: July 23, 2014 